\title{
NEW PROOFS OF THE THEOREMS OF BELTRAMI AND KASNER ON LINEAR FAMILIES
}

\author{
JOHN DECICCO
}

1. Introduction. We submit here new proofs, from a uniform point of view, of the theorems of Beltrami and Kasner on linear families of curves. Beltrami's result is that a surface $S$ may be mapped upon a plane $\pi$ so that its geodesics correspond to straight lines if and only if $S$ is of constant gaussian curvature [1]. ${ }^{1}$ Kasner's result states that a complete system of isogonal trajectories of a simple (that is, one-parameter) family of curves $F$ is linear if and only if $F$ is isothermal [2]. We shall also deduce from our work another theorem of Kasner stating that a surface $S$ can possess exactly $\infty^{2}$ isothermal families of geodesics (maximum possibility) if and only if $S$ is of constant gaussian curvature [3].

2. Velocity systems. For the development of our proofs, it is found necessary to consider certain classes of $\infty^{2}$ curves, namely, velocity systems, natural families, isogonal systems, $\Gamma_{0}$ and $\Gamma$ families. In Kasner's study of dynamical trajectories [4], an important class of $\infty^{2}$ curves was encountered which he termed velocity systems. In minimal coordinates $(u=x+i y, v=x-i y)$, any such system is defined by a second order differential equation of the form

$$
v^{\prime \prime}=v^{\prime}\left(c-d v^{\prime}\right),
$$

where $c$ and $d$ are arbitrary functions of $(u, v)$.

Special types of velocity systems are natural families and isogonal systems. Any natural family is a velocity system for which $c_{v}=d_{u}$, whereas any isogonal system is a velocity system for which $c_{v}=-d_{u}$.

A system of $\infty^{2}$ curves is both natural and isogonal if and only if it is the complete set of isogonal trajectories of an isothermal family. Such set is called a conformal rectilinear wex ${ }^{2}$ and is denoted by $\Gamma_{0}$. Any family of this type is conformally equivalent to the $\infty^{2}$ straight lines of the plane. A system $\Gamma_{0}$ is a velocity system for which $c_{v}=d_{u}$ $=0$.

Presented to the Society, October 31, 1942; received by the editors August 5, 1942.

${ }^{1}$ The numbers in brackets refer to the references at the end of the paper.

${ }^{2}$ The set of $\infty^{2}$ integral curves of any differential equation of second order $y^{\prime \prime}=F\left(x, y, y^{\prime}\right)$ has been termed a wex by Kasner. The transformation theory of $\Gamma_{0}$ systems has been developed by Kasner and DeCicco, Transformation theory of isogonal trajectories of isothermal families, Proc. Nat. Acad. Sci. U.S.A. 1942. 
3. The $\Gamma$ families. ${ }^{3}$ Any set of $\infty^{2}$ curves which is conformally equivalent to the set of $\infty^{2}$ circles orthogonal to a fixed proper circle (or a fixed straight line) is called a $\Gamma$ family. A $\Gamma_{0}$ system may be considered as the limiting case of a $\Gamma$ family by letting the radius of the fixed circle approach zero. The $\Gamma$ families are special types of velocity systems.

Theorem 1. A velocity system represents a $\Gamma$ family if and only if the functions $c$ and $d$ with $c_{v} \neq 0$ and $d_{u} \neq 0$ satisfy any one of the three equivalent systems of partial differential equations of second order

$$
\begin{gathered}
c_{u v}=c c_{v}, \quad c_{v v}=d c_{v} ; \\
d_{u v}=d d_{u}, \quad d_{u u}=c d_{u} ; \\
c_{v}=d_{u}, \quad c_{u v}=c c_{v}, \quad d_{u v}=d c_{v} .
\end{gathered}
$$

The last set of equations shows that every $\Gamma$ family is a natural family. But of course not every natural family is a $\Gamma$ family.

TheOrem 2. A $\Gamma$ family (including $\Gamma_{0}$ ) is a velocity system which contains exactly $\infty^{2}$ isothermal families.

A velocity system may contain exactly $\infty^{2}, \infty^{1}$, one, or no isothermal families. There are no other possibilities.

4. Proof of Kasner's linear characterization of isothermal families. We shall now state and prove Kasner's first theorem. A proof employing a complicated theorem on general linear families due to Lie and $\mathrm{R}$. Liouville, has been given elsewhere [2], but we shall prove it, beginning with basic principles. The result holds for the plane or any surface.

TheOREm 3. A simple (that is, one-parameter) family of curves is isothermal if and only if the complete set of its isogonal trajectories is a linear system.

Under any point transformation

$$
U=\phi(u, v), \quad V=\psi(u, v),
$$

with jacobian $J=\phi_{u} \psi_{v}-\phi_{v} \psi_{u} \neq 0$, the $\infty^{2}$ straight lines in the $(U, V)$ -

${ }^{3}$ It has been proved by Kasner that the only systems of $\infty^{2}$ circles which are given by second order differential equations of the cubic type $y^{\prime \prime}=A y^{\prime 3}+B y^{\prime 2}+C y^{\prime}$ $+D$, where $A, B, C, D$, are functions of $(x, y)$, are the $\Gamma$ and $\Gamma_{0}$ families. For additional properties of $\Gamma$ and $\Gamma_{0}$ families see Kasner and DeCicco, Geometry of velocity systems, Bull. Amer. Math. Soc. vols. 48-49 (1942-1943). 
plane correspond to the $\infty^{2}$ curves in the $(u, v)$-plane given by the differential equation of second order

$$
J v^{\prime \prime}=A v^{\prime 3}+B v^{\prime 2}+C v^{\prime}+D,
$$

where

$$
\begin{aligned}
& A=\phi_{v v} \psi_{v}-\phi_{v} \psi_{v v}, \quad D=\phi_{u u} \psi_{u}-\phi_{u} \psi_{u u}, \\
& B=\left(\phi_{v v} \psi_{u}-\phi_{u} \psi_{v v}\right)+2\left(\phi_{u v} \psi_{v}-\phi_{v} \psi_{u v}\right), \\
& C=\left(\phi_{u u} \psi_{v}-\phi_{v} \psi_{u u}\right)+2\left(\phi_{u v} \psi_{u}-\phi_{u} \psi_{u v}\right) .
\end{aligned}
$$

Now if (4) represents a velocity system, we must have $A=D=0$. We assume first both $\phi_{u}$ and $\phi_{v}$ not zero, and find

$$
\psi=a(u) \phi+b(u), \quad \psi=\alpha(v) \phi+\beta(v) .
$$

By substituting the first value of $\psi$ into $B$ and $J$, and then substituting the second value of $\psi$ into $C$ and $J$, we find that our functions $c$ and $d$ defining the velocity system (1) are given by

$$
c=\frac{\phi_{u u}}{\phi_{u}}-\frac{2 \phi_{u} \alpha_{v}}{\alpha_{v} \phi+\beta_{v}}, \quad d=\frac{\phi_{v v}}{\phi_{v}}-\frac{2 \phi_{v} a_{u}}{a_{u} \phi+b_{u}} .
$$

The equations (6) show that $\phi=(\beta-b) /(a-\alpha)$. Substituting this value of $\phi$ into (7), we find that any linear velocity system must be given by

$$
\begin{aligned}
& c=\frac{a_{u u}(\beta-b)+b_{u u}(a-\alpha)}{a_{u}(\beta-b)+b_{u}(a-\alpha)}-\frac{2\left(a_{u} \beta_{v}-\alpha_{v} b_{u}\right)}{\alpha_{v}(\beta-b)+\beta_{v}(a-\alpha)}, \\
& d=\frac{\alpha_{v v}(\beta-b)+\beta_{v v}(a-\alpha)}{\alpha_{v}(\beta-b)+\beta_{v}(a-\alpha)}-\frac{2\left(a_{u} \beta_{v}-\alpha_{v} b_{u}\right)}{a_{u}(\beta-b)+b_{u}(a-\alpha)} .
\end{aligned}
$$

Differentiating $c$ with respect to $v$ and $d$ with respect to $u$, and performing certain additions and subtractions, we find

$$
\begin{aligned}
c_{v}+2 d_{u} & =-\frac{3\left(a_{u u} b_{u}-a_{u} b_{u u}\right)\left[\alpha_{v}(\beta-b)+\beta_{v}(a-\alpha)\right]}{\left[a_{u}(\beta-b)+b_{u}(a-\alpha)\right]^{2}}, \\
2 c_{v}+d_{u} & =\frac{3\left(\alpha_{v v} \beta_{v}-\alpha_{v} \beta_{v v}\right)\left[a_{u}(\beta-b)+b_{u}(a-\alpha)\right]}{\left[\alpha_{v}(\beta-b)+\beta_{v}(a-\alpha)\right]^{2}}
\end{aligned}
$$

Now impose the condition $c_{v}=-d_{u}$ for isogonal systems. The addition of the preceding equations shows that

$$
\frac{\alpha_{v v} \beta_{v}-\alpha_{v} \beta_{v v}}{\left[\alpha_{v}(\beta-b)+\beta_{v}(a-\alpha)\right]^{3}}=\frac{a_{u u} b_{u}-a_{u} b_{u u}}{\left[a_{u}(\beta-b)+b_{u}(a-\alpha)\right]^{3}} .
$$


Assuming that the numerators of both of these fractions are not zero, the second order partial derivative with respect to $u$ and $v$ of the logarithm of this equation leads to the same equation but with the sign of one fraction changed. The addition and subtraction of this new equation and (10) prove that the numerators must be zero. This contradiction leads us to the fact that the numerators of both fractions in (10) must be zero.

Since the numerators of (10) are zero, it follows from (9) that $c_{v}=d_{u}=0$. Therefore the only possible linear isogonal systems are the $\Gamma_{0}$ families. That these are linear follows from the definition of $\Gamma_{0}$ systems. This completes the proof of Kasner's theorem in the general case. See $\$ 6$ for special case.

5. The linear natural families. Next we shall state and prove the following result. (This could also be deduced from Beltrami's theorem but our purpose here is to obtain it independently.)

Theorem 4. A natural family of curves is linear if and only if it is a $\Gamma$ or $\Gamma_{0}$ family.

By Theorem 3, we already know that a $\Gamma_{0}$ family is a linear natural family. Hence we can exclude this case, and we may assume that at least one of the first factors in the right-hand members of equations (9) is not zero.

By this last remark, it is found by taking the logarithmic derivative of the first of equations (9) with respect to $v$ and of the second with respect to $u$ that

$$
c_{v v}+2 d_{u v}=d\left(c_{v}+2 d_{u}\right), \quad 2 c_{u v}+d_{u u}=c\left(2 c_{v}+d_{u}\right) .
$$

Note that if either one of the first factors on the right-hand members of equations (9) is zero, the functions $c$ and $d$ must still satisfy the equations (11).

Now imposing the condition $c_{v}=d_{u}$ for a natural family, it is found by (11) and Theorem 1 that our linear natural family must be a $\Gamma$ family. Since $\Gamma$ and $\Gamma_{0}$ systems are linear natural, it is seen that Theorem 4 is proved in the general case. See $\$ 6$ for special case.

6. Proofs of Theorems 3 and 4 in the special cases. We shall prove our theorems for the case where $\phi_{v}=0$ and $\phi_{u} \neq 0$. The other case where $\phi_{u}=0$ and $\phi_{v} \neq 0$ is of course similar. For our special case, it is seen that in order that the differential equation (4) be a velocity system, the function $\psi$ must be given by

$$
\psi=\alpha(v) \phi+\beta(v) .
$$


The functions $c$ and $d$ defining the velocity system are then

$$
c=\frac{\phi_{u u}}{\phi_{u}}-\frac{2 \alpha_{v} \phi_{u}}{\alpha_{v} \phi+\beta_{v}}, \quad d=\frac{\alpha_{v v} \phi+\beta_{v v}}{\alpha_{v} \phi+\beta_{v}} .
$$

Differentiating the first with respect to $v$ and the second with respect to $u$, we find

$$
c_{v}=\frac{-2 \phi_{u}\left(\alpha_{v v} \beta_{v}-\alpha_{v} \beta_{v v}\right)}{\left(\alpha_{v} \phi+\beta_{v}\right)^{2}}, \quad d_{u}=\frac{\phi_{u}\left(\alpha_{v v} \beta_{v}-\alpha_{v} \beta_{v v}\right)}{\left(\alpha_{v} \phi+\beta_{v}\right)^{2}} .
$$

The condition $c_{v}=-d_{u}$ for an isogonal system, or the condition $c_{v}=d_{u}$ for a natural system shows that in either case we must have $c_{v}=d_{u}=0$. Therefore in our special case it is found that the only possible linear isogonal or linear natural systems are the $\Gamma_{0}$ families. This completes the proof of both Theorems 3 and 4 in the special cases.

7. Kasner's characterization of surfaces of constant curvature by isothermal families. Before proceeding with the proof of Kasner's second theorem, it is necessary to discuss some preliminary material. A natural family is a conformal image upon a plane of the geodesics of a general surface $S$. If $x=$ const. and $y=$ const. represent an isothermal net on $S$, the metric $d s$ for $S$ is given by

$$
d s^{2}=2 e^{\lambda(u, v)} d u d v .
$$

The natural family is then a velocity system for which

$$
c=\lambda_{u}, \quad d=\lambda_{v}
$$

The gaussian curvature $G$ of $S$ is $G=-e^{-\lambda} \lambda_{u v}$.

Now we state and prove the following result.

THEOREM 5. The conformal map of the geodesics of a surface $S$ upon a plane is a $\Gamma$ or $\Gamma_{0}$ family if and only if $S$ is of constant gaussian curvature. It is a $\Gamma_{0}$ family if and only if $S$ is developable.

The proof of this result is easily obtained by eliminating $c$ and $d$ from equations (2.3) and (16) and deducing from these two new equations that $G$ must be constant. Of course, $S$ is developable if and only if $\lambda$ is a harmonic function, and therefore if and only if a conformal image upon a plane of its geodesics is a $\Gamma_{0}$ family.

Now we shall state and prove Kasner's characterization of surfaces of constant curvature by isothermal families.

TheOREM 6. A surface $S$ can possess exactly $\infty^{2}$ isothermal families of geodesics if and only if $S$ is of constant gaussian curvature. 
For let a surface $S$ possess exactly $\infty^{2}$ isothermal families of geodesics. Our surface $S$ can be mapped upon a plane so that its geodesics are represented by a natural family which contains exactly $\infty^{2}$ isothermal families. By Theorem 2, the natural family must be a $\Gamma$ or $\Gamma_{0}$ system. From Theorem 5 , we see that $S$ must be of constant gaussian curvature.

The converse may be proved by the reversal of the argument of the preceding paragraph. Thus Theorem 6 is completely proved.

Kasner showed that the surfaces can be classified into three distinct types with respect to the number of isothermal families of geodesics: (1) the surfaces of constant curvature-exactly $\infty^{2}$; (2) the surfaces applicable to surfaces of revolution but of variable curvaature-exactly one, and (3) the surfaces not applicable to surfaces of revolution-none.

8. Beltrami's theorem. We shall now show how Beltrami's theorem may be deduced from our preceding work.

Theorem 7. A surface $S$ may be mapped upon a plane so that its geodesics correspond to straight lines if and only if $S$ is of constant gaussian curvature.

Let a surface $S$ be mapped upon a plane $\pi$ by a point transformation $T_{1}$ so that its geodesics correspond to straight lines. Now there exists a conformal transformation $T_{2}$ of $S$ upon $\pi$ so that the geodesics are represented by a natural family. Therefore the product $T_{1} T_{2}^{-1}$ carries the natural family into straight lines. By Theorem 4, the natural family must be a $\Gamma$ or $\Gamma_{0}$ system. Therefore by Theorem 5 , our surface $S$ must be of constant gaussian curvature.

Conversely, by the reversal of the steps involved in the preceding argument, it may be shown that a surface of constant curvature may be mapped upon a plane $\pi$ so that its geodesics correspond to straight lines. This completes the proof of Beltrami's Theorem 7.

\section{REFERENCES}

1. L. Bianchi, Vorlesungen ïber Differentialgeometrie, Leipzig, 1896.

2. E. Kasner, A characteristic property of isothermal systems, Math. Ann. vol. 59 (1904) pp. 352-354. Also a paper on Isothermal families to appear in the Rey Pastor volume edited by Beppo Levi, Rosario, Argentina, 1943.

3. - Isothermal systems of geodesics, Trans. Amer. Math. Soc. vol. 5 (1904) pp. 56-60.

4. —, Differential-geometric aspects of dynamics, Amer. Math. Soc. Colloquium Publications, vol. 3, 1912, 1934.

ILLINOIS INSTITUTE OF TECHNOLOGY 